\title{
Costos directos de atención médica en pacientes con diabetes mellitus tipo 2 en México: análisis de microcosteo
}

\author{
Rosibel de los Ángeles Rodríguez Bolaños, ${ }^{1}$ \\ Luz Myriam Reynales Shigematsu, ${ }^{1}$ Jorge Alberto Jiménez Ruíz, ${ }^{1}$ \\ Sergio Arturo Juárez Márquez ${ }^{2}$ y Mauricio Hernández Ávila ${ }^{3}$
}

Forma de citar

Rodríguez Bolaños RA, Reynales Shigematsu LM, Jiménez Ruíz JA, Juárez Márquez SA, Hernández Ávila M. Costos directos de atención médica en pacientes con diabetes mellitus tipo 2 en México: análisis de microcosteo. Rev Panam Salud Publica. 2010;28(6):412-20.

RESUMEN Objetivo. Estimar los costos directos de la atención médica a pacientes con diabetes mellitus tipo 2 (DM2) en el Instituto Mexicano del Seguro Social (IMSS).

Métodos. Se revisaron expedientes clinicos de 497 pacientes que ingresaron a unidades de segundo y tercer nivel de atención durante el período 2002-2004. Los costos se cuantificaron utilizando el enfoque de costeo de enfermedad (CDE) desde la perspectiva del proveedor, la técnica del microcosteo y la metodología de abajo-arriba (bottom-up). Se estimaron costos promedio anuales de diagnóstico, por complicación y total de la enfermedad.

Resultados. El costo total anual de los pacientes con DM2 para el IMSS fue de US\$452 064988 , correspondiente a 3,1\% del gasto de operación. El costo promedio anual por paciente fue de US\$3 193,75, correspondiendo US\$2 740,34 para el paciente sin complicaciones y US\$3 550,17 para el paciente con complicaciones. Los días/cama en hospitalización y en unidad de cuidados intensivos fueron los servicios con mayor costo.

Conclusiones. Los elevados costos en la atención médica a pacientes con DM2 y complicaciones representan una carga económica que las instituciones de salud deben considerar en su presupuesto, a fin de poder brindar un servicio de calidad, adecuado y oportuno. El empleo de la metodología de microcosteo permite un acercamiento a datos reales de utilización y manejo de la enfermedad.

Palabras clave Diabetes mellitus tipo 2; costo de enfermedad; atención médica; financiamiento en salud; México.

La diabetes mellitus (DM) es considerada un problema prioritario de salud

Instituto Nacional de Salud Pública (INSP), Morelos, México. La correspondencia se debe dirigir a Luz Myriam Reynales Shigematsu. Correo electrónico: 1reynales@insp.mx

2 Instituto Mexicano del Seguro Social (IMSS), Coordinación delegacional de investigación en salud, Morelos, México.

3 Subsecretaría de Prevención y Promoción de la Salud, Secretaría de Salud, México. pública global $(1,2)$. Se estima que para 2030 habrá cerca de 366 millones de diabéticos, de los cuales $70 \%$ residirán en países de ingresos medios y bajos $(1,3)$. Debido a su asociación con múltiples complicaciones microvasculares - retinopatía, nefropatía y neuropatía- y macrovasculares -infarto agudo de miocardio y enfermedades cerebrovascular y vascular periférica-, es considerada como un trastorno crónico complejo que conlleva a una disminución de la esperanza y de la calidad de vida en quienes la padecen (3). La DM constituye asimismo la cuarta causa de muerte por enfermedad, con una mortalidad que supera el millón de casos anuales, principalmente en los países en desarrollo (4). El tratamiento de las complicaciones, la invalidez temporal y permanente 
así como la mortalidad prematura que ocasiona representan una carga económica creciente en los sistemas de salud de todo el mundo $(5,6)$.

México se encuentra entre los primeros 10 países con mayor número de diabéti$\cos (3,7)$. Entre 1980 y 2000, la DM se convirtió en la primera causa de muerte en la población mexicana y durante los últimos cinco años su mortalidad ha crecido a un ritmo superior a 3\% anual (8). Según la Encuesta Nacional de Salud y Nutrición 2006 (ENSANUT 2006), la prevalencia nacional de diabetes por diagnóstico médico previo en adultos fue de $7,0 \%$ (7,3\% mujeres y 6,5\% hombres). Cabe destacar que dicha prevalencia se acentúa en función de la edad; así, para el grupo de 50 a 59 años, dicha proporción llegó a 13,5\% (14,2\% mujeres y 12,7\% hombres) y para el grupo de 60 a 69 años, $19,2 \%$ (21,3\% mujeres y 16,8\% hombres) (8). El primer censo de pacientes diabéticos, realizado en 2004 por el Instituto Mexicano del Seguro Social (IMSS), estimó un total de 2334340 personas afectadas por esta enfermedad. En ese mismo año se otorgaron 8,54 millones de consultas de medicina familiar para casos de diabetes, equivalentes a 13,0\% del total de consultas en esta especialidad (9). En el IMSS se registran anualmente cerca de 153000 nuevos casos de DM (420 diagnósticos diarios) y 18170 defunciones por sus complicaciones en individuos entre los 15 y los 64 años de edad (9).

La DM es, por ello, una enfermedad de alto costo para los sistemas de salud. Quienes la padecen acuden con mayor frecuencia a las unidades de atención médica, reciben más medicación, tienen una probabilidad mayor de ingresar a los servicios de urgencias y, debido a las múltiples complicaciones, requieren hospitalizaciones más prolongadas en comparación con los individuos que no son diabéticos (6). México, que no escapa a esta realidad, ha registrado fuertes incrementos en estos costos durante los últimos años. En 1992 los costos totales anuales de la enfermedad fueron estimados en alrededor de US\$ 430 millones, de los cuales US\$ 100 millones correspondieron a atención médica y US\$ 330 millones a pérdida de productividad y mortalidad prematura (10). Cálculos realizados en 2000 arrojaron totales anuales de US\$ 2618 millones, equivalentes a $15,5 \%$ del gasto total en salud y a $0,79 \%$ del producto interno bruto de ese año (11). En un análisis comparativo realizado en América Latina en 2003, los costos anuales derivados de DM tipos 1 y 2 en México ascendieron a US\$ 15118 millones, de los cuales US\$ 1974 millones correspondían a costos directos y US\$ 13144 millones a costos indirectos (12).

Arredondo y colaboradores estimaron los requerimientos financieros para atender la demanda de servicios durante tres años (2001-2003) de los pacientes con diabetes mellitus tipo 2 (DM2) en tres de las principales instituciones del sistema de salud pública mexicano: la Secretaría de Salud (SSA), el IMSS y el Instituto de Seguridad y Servicios Sociales de los Trabajadores del Estado (ISSSTE). Para el caso del IMSS, estimaron US \$83 millones por conceptos de atención hospitalaria y ambulatoria (13). Un estudio realizado en 2005 —en las mismas institucionesnotificó costos totales anuales de US\$ 317 millones, de los cuales US\$ 140 millones correspondieron a costos directos $\mathrm{y}$ US\$ 177 millones a costos indirectos. Cabe destacar que el costo total estimado exclusivamente para el IMSS fue de US\$ 103 millones (14). En México existen varios estudios con diferentes perspectivas y metodologías para calcular los costos derivados de la diabetes. En el presente trabajo se propuso estimar los costos directos de la atención médica a pacientes con diabetes mellitus tipo 2 (DM2) atendidos por el Instituto Mexicano del Seguro Social (IMSS).

\section{MATERIALES Y MÉTODOS}

\section{El Instituto Mexicano del Seguro Social}

El IMSS es la institución de seguridad social más grande en América Latina y representa un pilar fundamental para el bienestar individual y colectivo que brinda sus servicios a $44,0 \%$ de la población mexicana. En 2004 tenía una estructura normativa centralizada en cuatro regiones administrativas: Norte, Occidente, Centro y Sur, y contaba con 40 delegaciones en las diferentes entidades federativas del país. Existían 1340 unidades de atención médica (UAM) divididas en tres niveles de atención: 1076 unidades de medicina familiar (UMF, primer nivel de atención) donde se brindaba $85,0 \%$ de los servicios médicos; 223 hospitales regionales y generales de zona (segundo nivel) con 12,0\% de los servicios, y 41 unidades médicas de alta especialidad (UMAE, tercer nivel), donde se otorgaba
3,0\% del total de la atención médica. En ese mismo año había 42993343 adscritos al IMSS, de los cuales 37,8\% eran asegurados directos, principalmente trabajadores del sector privado; $52,5 \%$ eran familiares de asegurados; $5,1 \%$ pensionados, y $4,4 \%$ familiares de pensionados (15).

\section{Selección de las unidades de atención médica}

A través de un muestreo por conveniencia, se seleccionaron 12 delegaciones del IMSS para participar en este estudio. Se incluyeron todas las delegaciones en las que se ubicaba una unidad del tercer nivel de atención y dos delegaciones más que, a juicio experto, se consideraron representativas del segundo nivel de atención (hospital regional o general de zona). Las variables que se evaluaron para decidir la inclusión de las delegaciones fueron: el total de unidades médicas; el total de camas censables; la población adscrita, y el total de servicios ambulatorios y hospitalarios otorgados anualmente (16). La muestra final de unidades médicas quedó estructurada en cuatro regiones, 12 delegaciones y 28 UAM.

\section{Selección de la muestra de pacientes}

Una vez seleccionadas las UAM, se procedió a identificar los casos que podrían ser parte de la muestra. En una primera etapa, se partió de la población de los derechohabientes del IMSS que utilizaron los servicios de atención médica (ambulatoria, hospitalaria o ambas) en el período comprendido entre junio de 2002 y junio de 2004. Los casos de DM2 fueron identificados a partir del Sistema de Información Médica del IMSS (SIMO), utilizando la Clasificación Internacional de Enfermedades (CIE-10) (17). En una segunda etapa, se seleccionaron pacientes que recibieron atención médica por primera vez en el período señalado y que hubieran tenido un seguimiento mínimo de un año. Asimismo, debido a la metodología de costeo establecida, la muestra se redujo a los pacientes que cumplieran con los criterios recién mencionados y cuyos expedientes clínicos contaran con información completa, organizada, con notas de ingreso y egreso hospitalario, notas médicas y de enfermería, y con las hojas de procedimientos médicos, de laboratorio y de quirófanos. Finalmente, se seleccionaron 497 pacientes con diagnóstico confir- 
FIGURA 1. Modelo de costos de atención médica en el Instituto Mexicano del Seguro Social, México, 2004

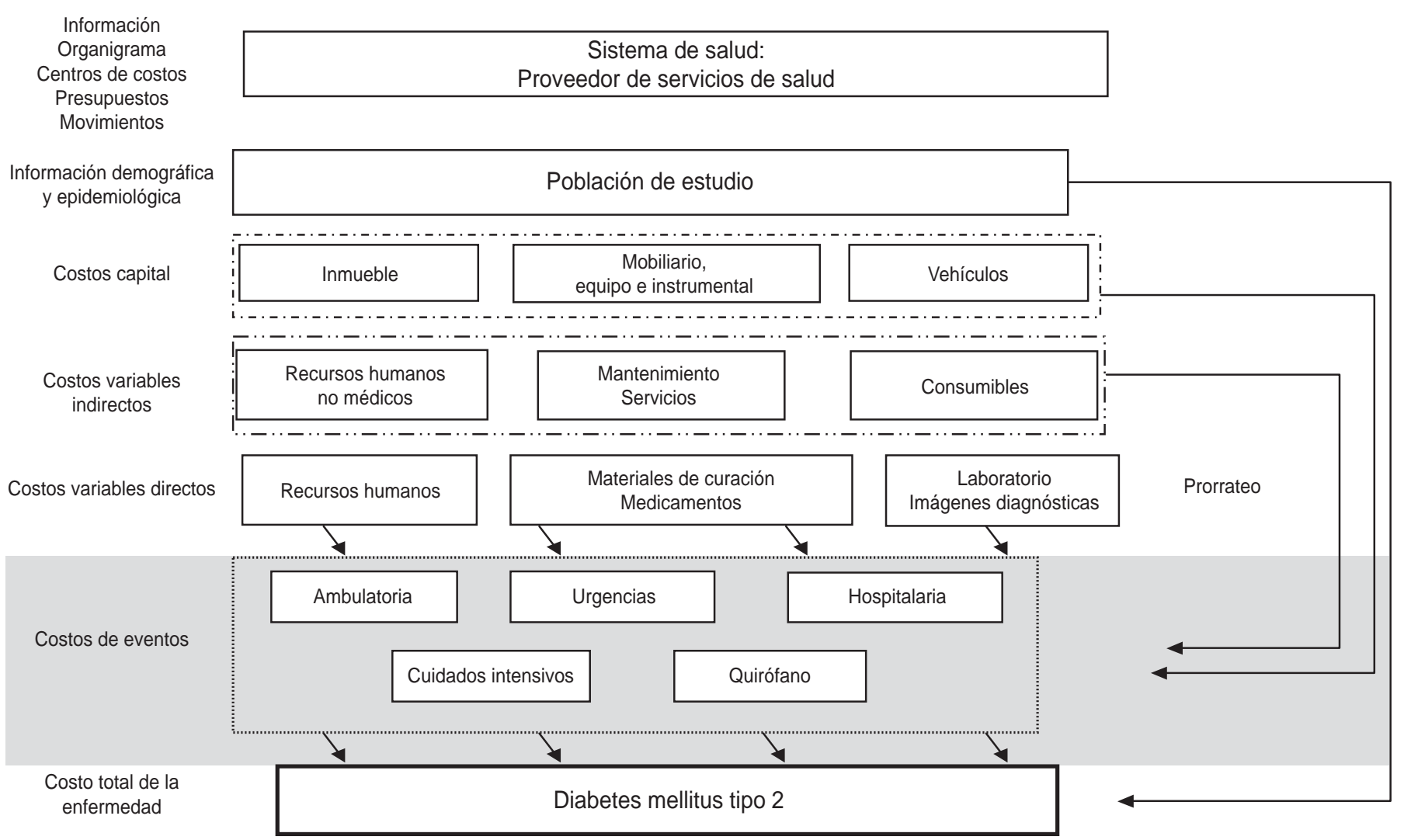

Fuente: elaboración de los autores.

mado de DM2, procurando que su distribución entre las UAM reflejara cierta proporcionalidad con la frecuencia de utilización de los servicios provistos.

Las complicaciones de la diabetes se establecieron de acuerdo con la CIE-10, considerándose así con DM2 sin complicaciones a aquellos pacientes diagnosticados con los códigos E11, E119, E14 y E149. En cuanto a quienes sí tenían complicaciones, se agruparon como renales las clasificaciones E111 y E141; oftálmicas E113 y E143, circulatorias periféricas E115 y E145, neurológicas E117 y E147, metabólicas E118 y E148 y con múltiples complicaciones E112 y E142. Los casos con complicaciones especificadas se clasificaron como E114 y E144, y los de complicaciones no especificadas como E116 y E146.

\section{Costos de la atención médica}

Los costos directos de la atención médica se calcularon desde la perspectiva del proveedor de los servicios de salud, considerando las cuatro regiones administrativas nacionales del IMSS y las uni- dades de primer, ${ }^{4}$ segundo ${ }^{5}$ y tercer ${ }^{6}$ nivel de atención médica de cada región. Se tomaron en cuenta los criterios y el enfoque de la metodología de costeo de enfermedad (CDE) basada en la prevalencia, que mide los costos actuales relacionados con la enfermedad (18). Esta metodología

\footnotetext{
4 El primer nivel de atención proporciona atención ambulatoria sin hospitalización, suministrada por los médicos familiares y profesionales de la salud (enfermeras y trabajadoras sociales) capacitados especialmente en servicios de medicina preventiva y curativa, así como en la promoción de la salud.

5 El segundo nivel de atención brinda atención médica en las cuatro especialidades básicas (pediatría, cirugía, medicina interna y ginecología y obstetricia), y algunas subespecialidades tales como cardiología, neurología y oncología clínica. Estos servicios reciben apoyo de las áreas de anestesiología, laboratorio clínico, rayos X, trabajo social, nutrición, banco de sangre, farmacia y ambulancia. Además, apoya el sistema de referencia y contrarreferencia de pacientes con los niveles primero y tercero.

6 El tercer nivel de atención proporciona atención médica de alta especialidad, como cardiología, hematología, neurología, neurocirugía, neumología, cirugía de tórax y oncología clínica y quirúrgica. Estos servicios reciben apoyo de las áreas de anestesiología, laboratorio clínico, rayos $\mathrm{X}$, trabajo social, nutrición, banco de sangre, farmacia y ambulancia. Además, apoya el sistema de referencia y contrarreferencia de pacientes con el segundo nivel de atención.
}

podía realizarse bajo dos enfoques: arriba-abajo (top-down) y abajo-arriba (bottom-up). En este estudio se empleó el segundo, que calcula los costos totales de enfermedad a partir de los costos unitarios de atención médica de tal manera que, primero, estima en forma detallada los costos de los procedimientos y tratamientos médicos, para luego agregarlos y obtener los costos totales de enfermedad.

Se desarrollaron modelos de costos unitarios considerando los componentes de costos fijos y variables que enfrentaban las unidades de segundo y tercer nivel de atención del IMSS (figura 1). Los costos de los materiales y medicamentos se obtuvieron con base en los precios promedio de compras de la institución para 2008. El costo de los recursos humanos se calculó a partir del salario integrado neto anualizado, tras considerar tanto las prestaciones sociales como otros beneficios no monetarios percibidos por el trabajador. Los costos de los activos fijos (inmuebles, equipo e instrumental médico) se depreciaron y prorratearon de acuerdo con la metodología de costo anual equivalente (CAE) $(19,20)$. 
La atención médica se clasificó en cinco eventos relevantes: a) ambulatoria; b) de urgencias; c) hospitalaria; d) intervención quirúrgica y e) en unidad de cuidados intensivos o unidad especializada. El costo total anual por paciente se estimó al considerar los costos unitarios de los eventos de atención médica y su frecuencia de utilización notificada en el año de seguimiento en el expediente clínico (ecuación 1).

Ecuación 1. Costo total paciente

$$
C T P_{j k w}=\sum_{i=1}^{n_{2}} Q R_{j k w i} \times P R_{i}
$$

Donde:

$\mathrm{CTP}_{j k w}$ : Costo para el paciente $k$, en evento médico j con DM2 en grado de severidad w

$Q R_{j k w i}$ : Utilización del recurso $i$ durante un año para la atención médica de un evento médico $j$ del paciente $k$ con DM2 en grado de severidad $w^{7}$

$P R_{i}$ : Precio o costo unitario del recurso $i$

w: Grado de severidad de la enfermedad

\{1. sin complicaciones, 2. con complicaciones\}

$k$ : Pacientes $\left\{1,2 \ldots n_{1}\right\}$

j: Tipo de evento médico \{1. Atención ambulatoria, 2. Atención de urgencias, 3. Hospitalización, 4. Cirugía, 5. Cuidados intensivos\}

$i$ : Recursos utilizados para la realización de la atención médica $\left\{1,2 \ldots n_{2}\right\}$

El costo promedio anual por paciente (CPA) se obtuvo con base en los costos totales anuales de todos los pacientes para cada una de las complicaciones de DM2. Finalmente, el costo total de la enfermedad (CTE) se obtuvo a través de un promedio ponderado de los costos de atención médica por cada una de las complicaciones por el número de casos registrados en el IMSS en cada subgrupo (ecuación 2).

\section{Ecuación 2. Costo total de la enfermedad}

$$
\begin{aligned}
C T E= & C P A_{\text {sincomplicaciones }} \times N_{\text {sincomplicaciones }}+ \\
& C P A_{\text {concomplicaciones }} \times N_{\text {concomplicaciones }}
\end{aligned}
$$

Donde:

CTE: Costo total de la enfermedad

CPA: Costo promedio anual por paciente en cada categoría

$N$ : Casos incidentes de enfermedad en cada categoría (21)

\footnotetext{
7 Los recursos utilizados por un paciente durante un año que se contabilizaron incluyeron materiales de curación, medicamentos, laboratorios e imágenes diagnósticas, y otros.
}

\section{RESULTADOS}

Del total de pacientes $(n=497)$, más de la mitad estuvo conformada por mujeres $(60,0 \%)$ y el promedio de edad fue de 61,4 años (desviación estándar [DE], 12). El nivel de escolaridad de poco menos de la mitad $(42,1 \%)$ fue de primaria, en tanto que $15,5 \%$ no contaba con ningún tipo de estudios. Por otra parte, solo $18,1 \%$ de la población de la muestra trabajaba al momento del estudio (cuadro 1). De acuerdo con la clasificación de complicaciones establecida en este estudio, $46,8 \%$ de los pacientes no presentaron complicaciones. Sin embargo, en los casos en que sí se presentaron, las complicaciones más frecuentes fueron las renales $(12,1 \%)$, seguidas de las oftálmicas (10,3\%), las circulatorias periféricas $(4,4 \%)$, las neurológicas $(2,0 \%)$ y las metabólicas $(0,9 \%)$. De los pacientes de la muestra, $11,5 \%$ tenían múltiples complicaciones, pero sólo en 10,0\% estaban especificadas. En cuanto a antecedentes patológicos, $15,6 \%$ de los pacientes

CUADRO 1. Características sociodemográficas de la población del estudio sobre costos de la diabetes mellitus tipo 2, Instituto Mexicano del Seguro Social (IMSS), México, 2002-2004

\begin{tabular}{lcr}
\hline $\begin{array}{l}\text { Características } \\
\text { sociodemográficas }\end{array}$ & No. de casos & $\%$ \\
\hline Sexo & & \\
Femenino & 299 & 60,2 \\
$\quad$ Masculino & 198 & 39,8 \\
Escolaridad & & \\
Ninguna & 77 & 15,5 \\
Primaria & 209 & 42,1 \\
Secundaria/técnica & 58 & 11,6 \\
Preparatoria/técnica & 29 & 5,8 \\
Profesional & 23 & 4,6 \\
Sin datos & 101 & 20,3 \\
Estado civil & & \\
Soltero(a) & 22 & 4,4 \\
Casado(a) & 298 & 60,0 \\
Unión consensual & 9 & 1,8 \\
Divorciado(a) & 16 & 3,2 \\
Viudo(a) & 75 & 15,1 \\
Separado(a) & 10 & 2,0 \\
Sin datos & 67 & 13,5 \\
Situación laboral & & \\
Trabaja & 90 & 18,1 \\
Quehaceres hogar & 197 & 39,6 \\
Jubilado/pensionado & 108 & 21,7 \\
Sin datos & 102 & 20,6 \\
Media de edad (DE) & & \\
& & \\
& &
\end{tabular}

Fuente: base de datos de microcosteo. Costos de atención médica atribuible al consumo de tabaco en el IMSS Nacional. Proyecto de investigación. México, 2004.

a Incluye población mayor de 35 años. DE: desviación estándar. con DM2 presentaban, adicionalmente, hipertensión esencial primaria. De los pacientes con complicaciones, $4,5 \%$ refirieron encontrarse en tratamiento con diálisis. Los medicamentos de mayor consumo registrado fueron glibenclamida $(15,8 \%)$, ácido acetilsalicílico $(9,0 \%)$, metformina $(5,2 \%)$, insulina $(5,1 \%)$.

El costo de la consulta y las pruebas de laboratorio para el diagnóstico confirmatorio de DM2, llevado a cabo por especialistas del segundo nivel de atención, ascendió a un promedio de US\$ 352 por paciente. En cuanto a la utilización de servicios de atención médica durante el primer año de seguimiento de los pacientes con DM2, se encontró que en promedio acudían a seis consultas ambulatorias e ingresaban a los servicios de urgencias dos veces al año, mientras que en los servicios de internación permanecían hospitalizados 10 días y en la unidad de cuidados intensivos, 6 días. Por otra parte, se sometían a intervenciones quirúrgicas dos veces al año. No se observaron diferencias en estos indicadores al comparar los pacientes con y sin complicaciones (cuadro 2). En relación con el servicio de atención ambulatoria, se halló que fue utilizado por menos de la mitad $(45,2 \%)$ de los pacientes con DM2; el costo anual promedio de este servicio fue de US\$ 480,5 (DE US\$ 2 059,4). La mayor presión de costos estuvo en el rubro de procedimientos, donde se estimó que fueron de US\$2 620,7 (DE US\$ 8 259,3). Los pacientes sin complicaciones acudieron con menos frecuencia $(19,7 \%)$ a la consulta ambulatoria en comparación con los pacientes con complicaciones (25,5\%). De los 208 pacientes que acudieron al servicio de urgencias, $55,0 \%$ lo hicieron por alguna complicación derivada de la DM2; el costo promedio por paciente se estimó en US\$ 582 (DE US\$ 449,4). Proporcionalmente los pacientes con complicaciones recurrieron con mayor frecuencia al servicio de urgencias. En términos de costo promedio no se observó una diferencia significativa entre ambos grupos de pacientes $(P<0,07)$ (cuadro 3).

Con respecto a los servicios de internación, 60,0\% de los pacientes hospitalizados tenían alguna complicación. Si bien el promedio de días / cama entre los pacientes con y sin complicaciones $(10 \mathrm{y}$ 9 días, respectivamente) no resultó estadísticamente significativo, hubo pacientes con estancias hospitalarias de 41 y 63 días en cada una de las categorías men- 
CUADRO 2. Utilización de los servicios de salud del Instituto Mexicano del Seguro Social (IMSS) durante el primer año de seguimiento por parte de pacientes con diabetes mellitus tipo 2, según hayan tenido o no complicaciones y por tipo de atención, México, 2002-2004

\begin{tabular}{|c|c|c|c|c|c|c|c|c|c|c|c|c|}
\hline \multirow[b]{2}{*}{ Atención } & \multicolumn{4}{|c|}{ Total } & \multicolumn{4}{|c|}{ Sin complicaciones ${ }^{a}$} & \multicolumn{4}{|c|}{ Con complicaciones ${ }^{b}$} \\
\hline & $n$ & No. & Media & Rango & $n$ & No. & Mediac $^{c}$ & Rango & $n$ & No. & Media $^{c}$ & Rango \\
\hline Ambulatoria & 1270 & 225 & 6 & $1-40$ & 571 & 98 & 6 & $1-16$ & 699 & 127 & 6 & $1-40$ \\
\hline Hospitalaria & 2559 & 259 & 10 & $1-63$ & 935 & 103 & 9 & $1-41$ & 1623 & 156 & 10 & $1-63$ \\
\hline Cuidados intensivos & 88 & 16 & 6 & $1-27$ & 43 & 6 & 7 & $1-27$ & 45 & 10 & 5 & $1-14$ \\
\hline Quirófano & 14 & 9 & 2 & $1-4$ & 5 & 4 & 1 & $1-2$ & 9 & 5 & 2 & $1-4$ \\
\hline
\end{tabular}

Fuente: base de datos de microcosteo. Costos de atención médica atribuible al consumo de tabaco en el IMSS Nacional. Proyecto de investigación. México, 2004.

Nota: $n$ se refiere a la frecuencia anual de uso de cada tipo de atención (ambulatoria y urgencias: consultas médicas o de urgencias; hospitalaria y cuidados intensivos: días de internación quirófano: procedimientos quirúrgicos) y No. al número de pacientes que utilizaron el servicio.

a Clasificación CIE-10 = E11, E119, E14 y E149 (17)

b Clasificación CIE-10 = E111, E141, E113, E143, E115, E145, E117, E147, E112, E142, E114, E144, E116 y E146 (17).

c Prueba $t$ : para cada uno de los servicios se realizó la prueba de igualdad de medias entre los grupos y en ningún caso se rechazó la hipótesis de que son iguales

CUADRO 3. Costo promedio anual de los servicios de salud utilizados por pacientes con diabetes mellitus tipo 2, según el tipo de atención médica recibida, Instituto Mexicano del Seguro Social (IMSS), México, 2002-2004

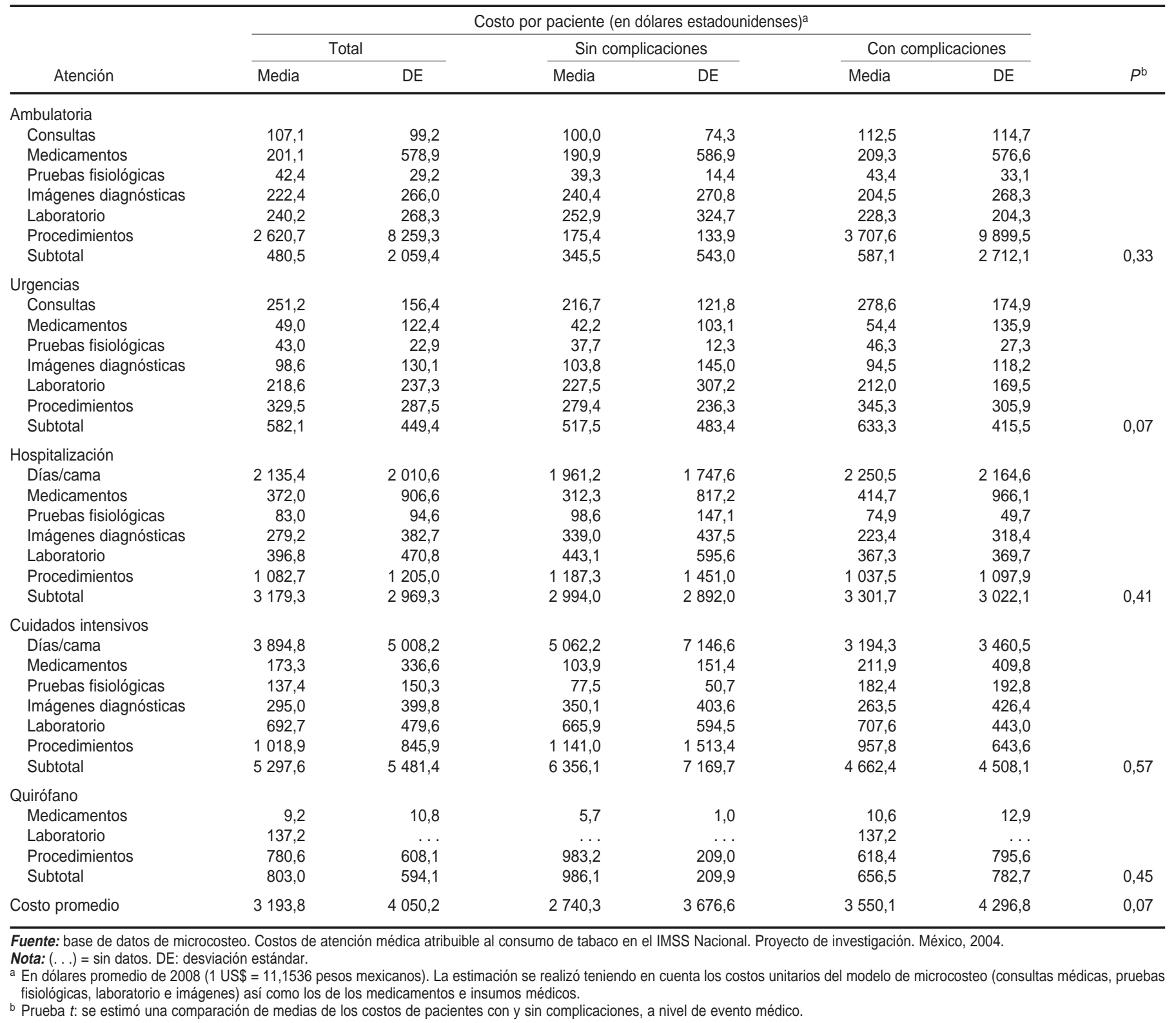


cionadas. El costo promedio estimado de US\$ 2 135,4 (DE US\$ 2 010,6) del día/ cama por paciente representó la mayor proporción de costos en este evento, sin diferencia significativa entre los grupos de pacientes con y sin complicaciones (cuadro 3). Los pacientes con complicaciones utilizaron los servicios de cuidados intensivos en mayor medida, con un costo promedio de US\$ 5297,6 (DE US\$ 5 481,4). Al igual que en el evento de hospitalización, la mayor presión de costos se observó en el día/cama. En total se registraron nueve procedimientos quirúrgicos, cinco de los cuales se realizaron en pacientes con complicaciones, y si bien el costo promedio de los pacientes sin complicaciones fue mayor (US\$ 986,1; DE US\$ 209,9) en comparación con lo ocurrido en el caso de los complicados (US\$ 656,5; DE US\$ 782,7), la diferencia no fue estadísticamente significativa (cuadro 3).

El costo promedio anual por paciente con DM2 fue de US\$ 3193,8 (DE US\$ 4 050,24), correspondiendo US $\$ 2740,34$ (DE US\$ 3676,66 ) para los pacientes sin complicaciones y US\$ 3550,17 (DE US\$ 4 296,78) para quienes sí las presentaron (cuadro 3). Es importante mencionar que los costos promedio de los eventos médicos no son resultado de la adición directa de sus componentes, ya que los pacientes no necesariamente reciben una atención médica estandarizada. De igual manera, los pacientes demandan de forma desigual los diferentes servicios de atención médica.

Para estimar los costos totales de la atención médica institucional, se consideró el total de pacientes con diagnóstico de DM2, de primer ingreso, atendidos durante 2008, lo que resultó en 142557 individuos (22). Los costos totales fueron ponderados de acuerdo con la proporción de pacientes con y sin complicacio-

CUADRO 4. Costo total anual de pacientes con diabetes mellitus tipo 2, según se hayan presentado o no complicaciones clínicas, Instituto Mexicano del Seguro Social (IMSS), México, 2002-2004

\begin{tabular}{lccc}
\hline & $\begin{array}{c}\text { Costo promedio } \\
\text { (en dólares estadounidenses) }^{\text {a }}\end{array}$ & $\begin{array}{c}\text { Costo total } \\
\text { No. de casos }\end{array}$ & \begin{tabular}{c} 
(en dólares estadounidenses) $^{\mathrm{a}}$ \\
\hline Sin complicaciones
\end{tabular} \\
Con complicaciones & 2740,3 & 66716 & 182821854,8 \\
Total & 3550,1 & 75841 & 269243134,1 \\
& NA $^{\mathrm{b}}$ & 142557 & 452064988,9
\end{tabular}

Fuente: estimaciones propias. Base de datos de microcosteo. Costos de atención médica atribuible al consumo de tabaco en el IMSS Nacional. Proyecto de investigación. México, 2004.

a Los costos se muestran en dólares promedio de 2008 ( 1 US $\$=11,1536$ pesos mexicanos) y el número de casos corresponde a los casos de incidencia en México para ese mismo año. Las complicaciones consideradas fueron: renales, oftálmicas, circulatorias periféricas, neurológicas, metabólicas, múltiples, especificadas y no especificadas.

b NA: datos no aplicables. nes notificada en este estudio y los costos estimados para cada categoría. El costo total anual de los pacientes con DM2 fue

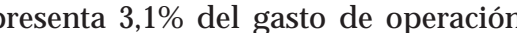
del IMSS.

\section{DISCUSIÓN}

En este estudio se examina el impacto económico que conlleva la atención médica de los pacientes con DM2 en el IMSS, la institución de seguridad social más de México. Los costos directos de nes, con US\$ 183 millones para los pacientes sin complicaciones y US\$269 millones para los pacientes con complicaciones.

A diferencia de los estudios previos realizados en el IMSS, en este trabajo se consideraron dos aspectos metodológicos importantes. Uno consistió en utilizar la metodología de CDE con el enfoque abajo-arriba, revisando una muestra de expedientes clínicos de las principales servar de de servicios médicos por parte de los pacientes diabéticos y la utilización, entre otros, de medicamentos, laboratorios y estudios de imágenes diagnósticas. Tal información es difícil de obtener a partir las metodologías utilizadas previafácil de implementar, no necesariamente reflejan la utilización real de los servicios de salud. Hay que señalar, sin embargo, que aun cuando la metodología promil a la demanda real de servicios y meDM, podría existir subnotificación en algunos rubros, como por ejemplo en el

.

\section{.} de US\$ 452064988 (cuadro 4), cifra que puesta se aproxima de forma más verosí- consumo de medicamentos, dada la forma en que se elabora parte de la historia clínica del paciente, en cuyo llenado generalmente participa también un tercero. De todas formas, esta probable limitación no invalida las tendencias o gradientes que pueden mostrar la utilización o los costos de los medicamentos, por lo que los montos calculados deben interpretarse como cifras conservadoras.

El otro aspecto metodológico relevante que se aplicó en este estudio fue la metodología de estimación de costos unitarios. Se diseñó un modelo de costos unitarios ad hoc para las unidades de atención médica del segundo y tercer nivel de atención del IMSS, que contemplaba los costos de capital, los costos variables directos e indirectos, el costo de los recursos humanos y las estrategias de prorrateo. Este modelo fue validado por las unidades contables de la institución y ofrece mayor precisión en la estimación de costos unitarios al compararlo con estimaciones realizadas mediante esquemas tarifarios o derivados de los gastos totales de las unidades de atención médica.

La disponibilidad de la utilización efectiva y los costos unitarios permitieron estimar los costos promedio de atención médica por paciente utilizando una metodología de microcosteo. Lo anterior significa un beneficio agregado para el IMSS, ya que esas estimaciones permiten identificar de manera precisa el sitio donde se localiza la mayor presión de costos de sus servicios de atención médica. En 2007, en los Estados Unidos de América se calcularon costos médicos directos de aproximadamente 27000 millones de dólares; de éstos, 50,0\% correspondían a costos por hospitalización, $12,0 \%$ a costos por suministro de medicamentos y $9,0 \%$ a costos por atención ambulatoria (23). En este estudio también se encontró que los servicios de internación en las áreas de hospitalización y cuidados intensivos, son los que presentan mayor presión de costos.

Los costos de internación se incrementan proporcionalmente de acuerdo tanto al número de ingresos en el año, como a los días de estancia institucional. Con respecto a esta última, el promedio fue de 10 días de hospitalización por paciente, cifra muy similar a la notificada para otras enfermedades crónicas - como el infarto de miocardio, la enfermedad pulmonar obstructiva crónica y el cáncer de pulmón- y mayor que el promedio de ocho días notificado para pa- 
cientes diabéticos en Argentina $(24,25)$. Si bien el tema rebasa los objetivos de este trabajo, cabe destacar que los hallazgos en los patrones de utilización de los servicios ambulatorios y de internación de los pacientes con DM2 no necesariamente son el reflejo del cumplimiento de las guías de atención médica establecidas en la institución o en la Norma Oficial Mexicana (NOM-015-SSA2-1994) (26).

Los costos estimados en el presente estudio sólo corresponden a pacientes con diagnóstico de DM2, el cual había sido confirmado por un especialista en hospitales correspondientes al segundo nivel de atención. Debido a las complicaciones que ya presentaban estos pacientes al momento de su diagnóstico, continuaron su tratamiento en hospitales y unidades médicas del segundo y tercer nivel de atención. No fue posible realizar el seguimiento de los pacientes en el primer nivel de atención, debido a la falta de integralidad del sistema de información del IMSS entre los niveles y entre las unidades de atención médica, lo cual representó el principal obstáculo para implementar la metodología de costeo en el primer nivel de atención. Por otra parte, este trabajo no contempla las estimaciones de los costos indirectos generados por incapacidad, invalidez y muerte pre- matura ocasionadas por DM, ya que al momento de realizarlo no se tuvo acceso a la información administrativa necesaria para realizar dicho análisis.

Cabe mencionar que en estudios internacionales se ha informado que el impacto económico de la atención médica a pacientes con DM es mayor en los países en desarrollo que en los desarrollados. Conforme a esos hallazgos, en América Latina y el Caribe los costos notificados para la atención médica de los pacientes con DM oscilan entre 2,5\% y 15,0\% del gasto total en salud, mientras que en los países desarrollados dichas tasas se ubican entre $5,0 \%$ y $6,0 \%(27,28)$.

En el caso de México, Villarreal y colaboradores estimaron que en 2000 el costo en atención médica para DM alcanzó unos US\$ 2618 millones, es decir $15,5 \%$ de su gasto total en salud y $0,79 \%$ de su PIB (11). Los costos directos de atención médica calculados en este estudio corresponden a 3,1\% del gasto total de operación que tuvo el IMSS en todo el país durante 2008. En el cuadro 5 se resumen los resultados de estudios realizados en México sobre los costos de la atención médica de la DM, donde se pueden apreciar las estimaciones en función de la metodología y de los conceptos de cuantificación.

\section{Conclusiones}

Se ha estimado que para 2025 en México habrá unos 11,7 millones de diabéticos, cifra que continuaría en aumento debido al envejecimiento, la urbanización y la alta prevalencia de obesidad e inactividad física que caracterizan a la población mexicana $(12,29)$. En consecuencia, se proyecta que para 2030 habrá un incremento desmedido en los costos de atención médica, estimados en US\$ 14695229000 y equivalentes a $15,0 \%$ del gasto total en salud, lo que ciertamente tendrá un impacto económico negativo en todo el sistema de salud nacional (6). En México la DM debe ser considerada como una prioridad de salud pública, cuyo manejo requiere informar a la sociedad en su conjunto acerca de la magnitud y la complejidad del problema. Asimismo, resulta imprescindible realizar investigación transdisciplinaria y asignar eficientemente los recursos en un modelo preventivo y de tratamiento intersectorial, integral y multifacético, como el establecido en 2004 en la Declaración de Acapulco (29).

En el caso particular del IMSS, es preciso realizar un análisis de minimización de costos que permita visualizar los posibles ahorros y lograr la redistribución más conveniente de los recursos hacia la pre-

CUADRO 5. Estudios realizados en el país sobre los costos de la atención médica de la diabetes mellitus, México, varios años

\begin{tabular}{|c|c|c|c|c|c|c|c|}
\hline \multirow[b]{2}{*}{ Estudio } & \multirow[b]{2}{*}{ Año } & \multirow[b]{2}{*}{ Metodología } & \multirow[b]{2}{*}{ Cobertura } & \multirow{2}{*}{$\begin{array}{l}\text { Año de } \\
\text { solicitud } \\
\text { financiera }\end{array}$} & \multicolumn{3}{|c|}{ Costos (en dólares estadounidenses) a } \\
\hline & & & & & Directos & Indirectos & Totales \\
\hline Phillips M, Salmeron J (10) & 1992 & & Nacional & & 99936000 & 330000000 & 429936000 \\
\hline Villareal-Ríos et al. (11) & 2000 & & Nacional & & 2618000000 & $\ldots$ & . \\
\hline Barceló A et al. (12) & 2003 & $\begin{array}{l}\text { Basado en prevalencia } \\
\text { Encuestas nacionales y } \\
\text { estudios de diferentes países }\end{array}$ & Nacional & 2000 & 1974200000 & 13144100000 & 15118200000 \\
\hline \multirow[t]{2}{*}{ Arredondo A (13) } & 2001 & $\begin{array}{l}\text { Técnica de instrumentación } \\
\text { Consenso manejo de caso } \\
\text { promedio }\end{array}$ & IMSS & $\begin{array}{l}2001 \\
2002\end{array}$ & $\begin{array}{l}\cdots \\
\cdots\end{array}$ & $\cdots$ & $\begin{array}{l}76769795 \\
77865649\end{array}$ \\
\hline & & & & 2003 & 83659227 & $\cdots$ & 79086723 \\
\hline \multirow[t]{2}{*}{ Arredondo A, Zuniga A (14) } & 2004 & $\begin{array}{l}\text { Técnica de instrumentación } \\
\text { Consenso manejo de caso promedio }\end{array}$ & $\begin{array}{l}\text { Nacional } \\
\text { IMSS }\end{array}$ & 2005 & $\begin{array}{r}140410816 \\
83748439\end{array}$ & $\begin{array}{l}177220390 \\
107134006\end{array}$ & $\begin{array}{l}317631206 \\
190882445\end{array}$ \\
\hline & & Perspectiva proveedor & $\begin{array}{l}\text { SSA } \\
\text { ISSSTE }\end{array}$ & & $\begin{array}{l}37079588 \\
19582796\end{array}$ & $\begin{array}{l}45607891 \\
24478493\end{array}$ & $\begin{array}{l}82687479 \\
44061289\end{array}$ \\
\hline Presente estudio & & $\begin{array}{l}\text { Costeo de la enfermedad } \\
\text { Abajo-arriba-perspectiva proveedor }\end{array}$ & IMSS & 2008 & 452064988 & $\cdots$ & $\cdots$ \\
\hline \multirow[t]{2}{*}{ Zhang $P$, et al. (6) } & 2010 & $\begin{array}{l}\text { Simulación basada en el gasto } \\
\text { en salud de pacientes diabéticos }\end{array}$ & & & & & \\
\hline & & vs. no diabéticos & Nacional & 2010 & 4836480000 & $\ldots$ & $\ldots$ \\
\hline
\end{tabular}

Fuente: elaboración de los autores.

Nota: Abreviaturas. (. . .): sin datos. IMSS: Instituto Mexicano del Seguro Social. SSA: Secretaría de Salud, México. ISSSTE: Instituto de Seguridad y Servicios Sociales de los Trabajadores del Estado, México.

a En dólares estadounidenses de 2008 ( 1 US $\$=11,1536$ pesos mexicanos). 
vención y el mejor manejo médico de las etapas tempranas de la enfermedad. El beneficio potencial en términos económicos puede obtenerse con medidas preventivas de educación enfocadas a la población general, con el fin de implementar estilos de vida saludables y disminuir así la incidencia de pacientes con DM2 (30-32). Con esta idea, es prioritario reforzar la capacitación del personal de salud de las unidades y hospitales de primer y segundo nivel de atención a fin de: 1) poner en práctica las estrategias de tamizaje e identificar a la población en riesgo, especialmente aquellos individuos que presentan el síndrome metabólico; 2) efectuar un diagnóstico acertado, y 3) promover un tratamiento individualizado efectivo orientado a la prevención/retraso de la aparición de las complicaciones, con estricto apego y cumplimiento a lo establecido en las guías de atención médica y en la NOM-015-SSA21994 (26). Dado que hoy en día la diabetes aparece en poblaciones cada vez más jóvenes, el IMSS podría aprovechar esta ventana de oportunidad para echar a andar programas preventivos en las empresas afiliadas a la institución.

Para abatir y controlar la epidemia de diabetes es importante que el IMSS pro- mueva la investigación interdisciplinaria de esta enfermedad. Tal emprendimiento implica contar con un sistema de información integrado - como ya ha sido propuesto- que permita identificar a la población en riesgo, generar un censo de pacientes diabéticos, evaluar los tratamientos de manera individual y medir el impacto de las intervenciones establecidas institucionalmente (33). Esta información permitirá definir las acciones necesarias para superar los grandes retos en el control individual de la DM, como son la baja adherencia al tratamiento y el pobre cumplimiento de las recomendaciones dietéticas y de actividad física, además de las deficiencias institucionales en cuando a la oferta de servicios y disponibilidad de medicamentos orientados al control glicémico.

Finalmente, es importante que en este sistema de información estén integradas las áreas administrativo-financieras (afiliación, prestaciones económicas, contabilidad), clínicas (laboratorio e imágenes diagnósticas) y de abastecimiento y farmacia, de tal manera que se cuente con un registro tanto de su utilización efectiva como de sus costos unitarios. Disponer de esta información permitiría com- plementar las estimaciones de costos en las etapas tempranas de la enfermedad $\mathrm{y}$, en las unidades del primer nivel de atención, realizar análisis de costo-efectividad y de evaluación de impacto de las diferentes estrategias de intervención orientados a fortalecer el proceso de toma de decisiones por parte de las autoridades institucionales.

Agradecimientos. Esta investigación fue posible gracias al financiamiento del Consejo Nacional de Ciencia y Tecnología y del Instituto Mexicano de Seguro Social. Los autores agradecen a los coordinadores de investigación en funciones durante 2004 en las delegaciones del IMSS de Nuevo León, Chihuahua, San Luis Potosí, Jalisco, Guanajuato, Nayarit, Distrito Federal, Estado de México, Guerrero, Puebla, Tabasco y Chiapas, así como a las personas que participaron en el levantamiento, codificación y recogida de la información. También desean expresar especial gratitud a Homero Martínez Salgado, entonces coordinador de investigación del IMSS, por todo el apoyo brindado para la concreción del presente trabajo.

\section{REFERENCIAS}

1. Wild S, Roglic G, Green A, Sicree R, King H. Global prevalence of diabetes. Estimates for the year 2000 and projections for 2030. Diabetes Care. 2004;27:1047-53.

2. King H, Aubert RE, Herman WH. Global burden of diabetes, 1995-2025. Prevalence, numerical estimates, and projections. Diabetes Care. 1998;21(9):1414-31.

3. International Diabetes Federation (IDF). Diabetes Atlas. 4. ${ }^{\text {a }}$ ed. Brussels: IDF; 2009.

4. World Health Organization. Fact Sheet No. 312. Diabetes. Hallado en: http://www.who. int/mediacentre/factsheets/fs312/en/. Acceso el 14 de noviembre de 2010.

5. Ettaro L, Songer TJ, Zhang P, Engelgau MM. Cost-of-illness studies in diabetes mellitus. Pharmacoeconomics. 2004;22:149-64.

6. Zhang P, Zhang X, Brown J, Vistien D, Sicree $\mathrm{R}$, Shaw J, et al. Global healthcare expenditures on diabetes for 2010 and 2030. Diabetes Res Clin Pract. 2010;87:293-301.

7. Martorell R. La diabetes y los mexicanos: ¿Por qué están vinculados? Prev Chronic 2005 Jan. Hallado en: http://www.cdc.gov/pcd/ issues/2005/jan/040100es.htm. Acceso el 14 de noviembre de 2010.

8. Olaiz-Fernández G, Rivera Dommarco J, Shamah Levy T, Rojas R, Villalpando Hernández S, Hernández Ávila M, et al. Encuesta Nacional de Salud y Nutrición 2006. Cuernavaca, México: Instituto Nacional de Salud Pública; 2006.
9. Fernández Cantón S. El IMSS en cifras: El censo de pacientes diabéticos, 2004. Rev Med Inst Mex Seguro Soc. 2006;44(4):375-82.

10. Phillips M, Salmeron J. Diabetes in Mexico: A serious growing problem. World Health Stat Q. 1992;45:338-46.

11. Villarreal Rios E, Salinas Martinez AM, Medina Jauregui A, Garza Elizondo ME, Nunez Rocha G, Chuy Diaz ER. The cost of diabetes mellitus and its impact on health spending in Mexico. Arch Med Res. 2000;31(5):511-4.

12. Barceló A, Aedo C, Rajpathak S, Robles S. The cost of diabetes in Latin America and the Caribbean. Bull World Health Organ. 2003; 81(1): 19-27.

13. Arredondo A. Requerimientos financieros para la demanda de servicios de salud por diabetes e hipertensión. Rev Invest Clin. 2001; 53(5):422-9.

14. Arredondo A, Zuniga A. Economic consequences of epidemiological changes in diabetes in middle-income countries. The Mexican case. Diabetes Care. 2004;27(1):104-9.

15. Instituto Mexicano del Seguro Social. Coordinación de Afiliación al Régimen Obligatorio. Hallado en: http://www.imss.gob.mx/ estadisticas/financieras/cubo.htm. Acceso el 22 de noviembre 2010.

16. Secretaría de Salud, México. Información básica sobre recursos del Sistema Nacional de Salud 2001. Salud Publica Mex. 2002;44:476-85.
17. Organización Mundial de la Salud. Clasificación estadística internacional de enfermedades y problemas relacionados con la salud. 10. ${ }^{\text {a }}$ revisión. v. 3. Lista tabular. Washington, D.C.: OMS; 1995.

18. Single E, Collins D, Easton B, Harwood H, Lapsley H, Kopp P, et al. International Guidelines for Estimating the Costs of Substance Abuse-Second Edition. Geneva: World Health Organization; 2003.

19. Drummond M, O'Brien B, Stoddart G, Torrance G. Métodos para la evaluación económica de los programas de asistencia sanitaria. 2. ${ }^{\mathrm{a}}$ ed. Madrid: Ediciones Dáz de Santo; 2001.

20. Castaño-Convers S. Finanzas y costos. Un acercamiento a la gestión financiera de las organizaciones de salud. Bogotá, Colombia: Centro de Gestión Hospitalaria; 2002.

21. Stata 9. 1984-2001, Stata Corporation College Station: Texas, USA.

22. Instituto Mexicano del Seguro Social, México. Dirección de Prestaciones Médicas, Unidad de Salud Pública. Coordinación de vigilancia epidemiológica y apoyo en contingencias. Boletín semanal de vigilancia epidemiológica. México: Instituto Mexicano del Seguro Social; 2008.

23. American Diabetes Association. Economic cost of diabetes in the US in 2007. Diabetes Care. 2008;31(3):596-615.

24. Reynales Shigematsu LM, Rodríguez Bolaños RA, Jiménez JA, Juárez Márquez SA, Castro 
Ríos A, Hernández Ávila M. Costos de la atención médica atribuibles al consumo de tabaco en el Instituto Mexicano del Seguro Social. Salud Publica Mex. 2006;48(1):48-64.

25. Caporale JE, Calvo H, Gagliardino JJ. Costos de atención médica de personas con diabetes anteriores y posteriores a su hospitalización en Argentina. Rev Panam Salud Publica. 2006; 20(6):361-8

26. Norma Oficial Mexicana NOM-015-SSA2-1994. Modificación a la norma para la prevención, tratamiento y control de la diabetes mellitus en la atención primaria. Hallado en: http:// www.salud.gob.mx/unidades/cdi/nom/ m015ssa24.html. Acceso el 14 de noviembre de 2010.

27. Venkat Narayan KM, Zhang P, Kanaya AM, Williams DE, Engelgau MM, Imperatore G, et al. Diabetes: The pandemic and potential solutions. En: Jamison DT, Breman JG, Measham AR, Alleyne G, Claeson M, Evans DB, et al., eds. Disease control priorities in developing countries. USA: Oxford University Press/The World Bank; 2005. Pp. 591-603.

28. American Diabetes Association. Economic consequences of diabetes mellitus in the U.S. in 1997. Diabetes Care. 1998;21(2):296-309.

29. Aguilar-Salinas C, Gómez-Pérez FJ. Declaración de Acapulco: Propuesta para la reducción de la incidencia de diabetes en México. Rev Invest Clin. 2006:58;1:71-7

30. Gagliardino JJ, Etchegoyen G. A model educational program for people with type 2 diabetes: a cooperative Latin American implementation study (PEDNID-LA). Diabetes Care. 2001;24(6):1001-7.
31. Khuwaja AK, Khowaja LA, Cosgrove P. The economic cost of diabetes in developing countries: some concerns and recommendations. Diabetologia. 2010;53:389-90.

32. Kogan AJ. Overcoming obstacles to effective care of type 2 diabetes. Report Am J Manag Care. 2009;15:S255-62

33. Arredondo A, De Icaza E. Financial requirements for the treatment of dibetes in Latin America: implications for the health system and for patients in Mexico. Diabetologia. 2009:52:1693-5.

Manuscrito recibido el 25 de enero de 2010. Aceptado para publicación, tras revisión, el 19 de octubre de 2010.

ABSTRACT Objective. Estimate the direct cost of medical care incurred by the Mexican Social Security Institute (IMSS, Instituto Mexicano del Seguro Social) for patients with type 2 diabetes mellitus (DM2).

Direct costs of medical care for patients with type 2 diabetes mellitus in Mexico: micro-costing analysis

Methods. The clinical files of 497 patients who were treated in secondary and tertiary medical care units in 2002-2004 were reviewed. Costs were quantified using a disease costing approach (DCA) from the provider's perspective, a micro-costing technique, and a bottom-up methodology. Average annual costs by diagnosis, complication, and total cost were estimated.

Results. Total IMSS DM2 annual costs were US\$452 064988 , or 3.1\% of operating expenses. The annual average cost per patient was US\$3 193.75, with US\$2 740.34 per patient without complications and US\$3 550.17 per patient with complications. Hospitalization and intensive care bed-days generated the greatest expenses.

Conclusions. The high cost of providing medical care to patients with DM2 and its complications represents an economic burden that health institutions should consider in their budgets to enable them to offer quality service that is both adequate and timely. Using the micro-costing methodology allows an approximation to real data on utilization and management of the disease.

Key words Diabetes mellitus, type 2; cost of illness; medical care; financing, health; Mexico. 